\title{
Existence and monotonicity of solutions to moral hazard problems
}

\author{
G. Carlier \\ Université Paris Dauphine, CEREMADE, UMR CNRS 7534, Place du Maréchal \\ De Lattre De Tassigny 75775 PARIS CEDEX 16 \\ R.-A. Dana \\ Université Paris Dauphine, CEREMADE, UMR CNRS 7534, Place du Maréchal \\ De Lattre De Tassigny 75775 PARIS CEDEX 16
}

\begin{abstract}
This paper provides a method to prove existence of solutions to some moral hazard problems with infinite set of outcomes. The argument is based on the concept of nondecreasing rearrangement and on a supermodular version of Hardy-Littlewood's inequality. The method also provides qualitative properties of solutions. Both the cases of wage contracts and of insurance contracts are studied.
\end{abstract}

Key words: moral hazard, first-order approach, rearrangements, supermodularity.

\section{Introduction}

Since Mirrlees' early work [Mirrlees (1975)], the principal-agent problem when there is moral hazard has received a lot of attention. Under the assumption that the agent chooses an action from a real interval, a first line of research on the subject has focused on the so-called "first-order approach" (see [Holmstrom (1975)], [Jewitt (1988)], [Rogerson (1985)]) which involves relaxing the constraint that the agent chooses an action which is utility maximizing to require only that the agent chooses an action at which his utility is a stationary point. Rogerson [Rogerson (1985)] and Jewitt [Jewitt (1988)] have given sufficient conditions for the first-order approach to be valid. By this,

Email addresses: carlier@ceremade.dauphine.fr (G. Carlier), dana@ceremade.dauphine.fr. (R.-A. Dana). 
they mean that, the original problem (that we shall from now on call the second-best problem) and the relaxed problem have the same solutions (without taking into account extreme efforts). Rogerson [Rogerson (1985)] and Jewitt [Jewitt (1988)] prove the monotonicity of the optimal solutions of the relaxed problem, a fundamental result for the validity of the first-order approach and deduce the monotonicity of second-best solutions (modulo extreme efforts). However, their results depend heavily on whether there exist solutions to the second-best problem and to the relaxed problem. This is by no mean a restriction if the set of outcomes is finite since under mild continuity assumptions on the datas of the model, there exist solutions to these problems. This fact has been noticed by Grossman and Hart [Grossman and Hart (1983)] who under the assumption that there were a finite number of outcomes, proved directly the existence of solutions to the second-best problem and gave sufficient conditions for their monotonicity using first-order arguments.

When the set of outcomes is infinite, a standard weak-compactness argument may not be applied to prove existence of solutions because of the lack of convexity (hence of weak upper semicontinuity) of the problem studied. A second line of research, starting with Page [Page (1987)] has developed an existence theory to principal-agents problems, in a general abstract framework. Existence is proven under mild topological assumptions provided that the set of admissible contracts fulfills, ex ante, some compactness property. In particular, Page ([Page (1987)], [Page (1991)]) gives several examples of contract sets containing optimal contracts, including the case of bounded monotone contrats.

Our paper lies in between these two streams of reseach. As in [Balder (1996)], [Page (1987)], and [Page (1991)], we deal with the issue of existence of secondbest solutions. Our framework is that of [Jewitt (1988)] and [Rogerson (1985)] with a continuum of monetary outcomes and we also use the first-order approach. It is well-known that, under the monotone likelihood ratio hypothesis (MLRC), the optimal solutions to the relaxed problem, if they exist, are monotone. This fact in itself, however, does not allow to restrict the study of the relaxed problem to nondecreasing admissible contracts. Our specific contribution is to show that under (MLRC), any admissible contract for the relaxed problem is dominated by a nondecreasing admissible contract. This is obtained by using the concept of nondecreasing rearrangement and on a supermodular version of Hardy-Littlewood's inequality. This property allows us to assume without loss of generality, that admissible contracts are nondecreasing and to obtain existence of solutions to the relaxed problem by a standard compactness argument (Helly's theorem). We then use the convexity of the distribution function hypothesis (CDFC) to prove existence and monotonicity (modulo boundary cases) of second-best contracts. While the MLRC hypothesis is essential to our rearrangement argument, the CDFC hypothesis 
is only used for sake of simplicity.

We study two cases:

- wage contracts which are assumed to be unconstrained and such that the observation of a higher level of output allows the inference that the agent worked harder in the sense of stochastic dominance,

- insurance contracts that are assumed to be constrained by liabilities and such that the observation of a higher loss allows the inference that the agent did less effort in the sense of stochastic dominance.

In section 2, we recall a standard model of wage contracts with moral hazard with a real interval of actions and an infinite number of outcomes. The secondbest problem is introduced as well as some assumptions. Section 3 is devoted to the study of a relaxation of the second-best problem. Using rearrangement techniques, we first prove that any contract admissible for the relaxed problem is dominated by a nondecreasing admissible contract and that without loss of generality, admissible contracts for the relaxed problem may be assumed to be nondecreasing. We then deduce existence of optimal solutions to the doublyrelaxed problem. In section 4, combining those results with standard results in the first-order approach literature, we prove existence of second-best contracts and fully characterize them. Section 5 is devoted to the special case of a riskneutral principal and section 6 to that of insurance contracts.

\section{The model}

We consider a standard principal-agent model with moral hazard with a continuum of actions and a continuum of outcomes. Agents choose an effort $e$ from $\mathbb{R}_{+}$that is unobservable to the principal. The outcome $x$ belongs to the given segment $[0, \bar{x}]$. The probability law of outcome when the agent's effort is $e$ is given by a density $f(x, e)$. Let $w(x)$ be the wage paid to the agent if outcome $x$ occurs.

The agent's utility function is assumed to be von Neumann-Morgenstern and quasi-linear:

$$
U(w, e):=\int_{0}^{\bar{x}} u(w(x)) f(x, e) d x-e .
$$

The principal's utility function is von Neumann-Morgenstern:

$$
V(w, e):=\int_{0}^{\bar{x}} v(x-w(x)) f(x, e) d x .
$$


A contract $(w, e)$ is incentive-compatible if:

$$
U(w, e)=\max _{e^{\prime} \geq 0} U\left(w, e^{\prime}\right)
$$

and individually rational if:

$$
U(w, e) \geq \underline{u}
$$

where $\underline{u}$ is a minimum expected utility level the agents must be guaranteed.

The principal's second-best problem then reads as:

$$
\sup \{V(w, e): e \geq 0,(w, e) \text { satisfies }(3) \text { and }(4)\} .
$$

We introduce the following assumptions on the data.

(H1): The utility functions $u$ and $v: \mathbb{R} \rightarrow \mathbb{R}$ are strictly concave, increasing, twice continuously differentiable, and there exists $\alpha \in(0,1)$ such that

$$
\lim _{y \rightarrow+\infty} \frac{u(y)}{y^{\alpha}}=\lim _{y \rightarrow+\infty} \frac{v(y)}{y^{\alpha}}=0 .
$$

(H2): $(i)$ The density function $f(.,$.$) is continuous on [0, \bar{x}] \times \mathbb{R}_{+}$, (ii) there exist constants $b>a>0$ such that $f(x, e) \in[a, b]$ for all $(x, e)$,

(iii) for all $x, f(x,$.$) is twice continuously differentiable and the first and sec-$ ond partial derivatives of $f$ with respect to $e, f_{e}$ and $f_{e e}$ are jointly continuous and bounded on $[0, \bar{x}] \times \mathbb{R}_{+}$.

As in [Rogerson (1985)], let us introduce the relaxed condition:

$$
U_{e}(w, e)=\int_{0}^{\bar{x}} u(w(x)) f_{e}(x, e) d x-1 \geq 0 .
$$

Let $A$ be the set of pairs $(w, e)$ that fulfill (4) and (5):

$$
A:=\{(w, e): e \geq 0,(w, e) \text { satisfies (4) and (5) }\} .
$$

(H3): The set $A$ is nonempty and there exists $(w, e) \in A$ such that $V(w, e)>$ $-\infty$.

(MLRC): For all $e \geq 0$, the function $L R(.,):. x \mapsto L R(x, e):=f_{e}(x, e) / f(x, e)$ is nondecreasing on $[0, \bar{x}]$.

We shall sometimes use the stronger assumption:

(MLRCstrong): For all $e \geq 0$, the function $L R(.,):. x \mapsto L R(x, e):=$ $f_{e}(x, e) / f(x, e)$ is increasing on $[0, \bar{x}]$. 


\section{The doubly-relaxed problem}

Following [Rogerson (1985)], we define the principal's doubly-relaxed problem as:

$$
\sup \{V(w, e),(w, e) \in A\} .
$$

This section is devoted to the study of this program. We shall prove existence and monotonicity of solutions. This will follow from rearrangement inequalities that we recall in the next paragraph.

\subsection{Rearrangements and supermodularity}

For the proofs of the results given in this paragraph and some applications, we refer the interested reader to [Lorentz (1953)], see also [Carlier and Dana (2002)] and the references listed therein.

Let $\mu$ be a probability measure on $[0, \bar{x}]$. Assume that $\mu$ is absolutely continuous with respect to Lebesgue's measure and that it admits a continuous and positive density $f$ on $[0, \bar{x}]$.

Definition-Property 1 Two Borel functions on $[0, \bar{x}], v$ and $w$ are equimeasurable with respect to $\mu$ if and only if they fulfill one of the following equivalent conditions:

(1) $\mu\left(v^{-1}(B)\right)=\mu\left(w^{-1}(B)\right)$ for every Borel subset $B$ of $\mathbb{R}$,

(2) for every bounded continuous function $g$ :

$$
\int_{0}^{\bar{x}} g(v(x)) d \mu(x)=\int_{0}^{\bar{x}} g(w(x)) d \mu(x)
$$

Proposition 1 Let $w$ be any real-valued Borel function on $[0, \bar{x}]$. Then there exists a unique right-continuous nondecreasing function, $\widetilde{w}$, which is equimeasurable to $w$.

In the previous statement $\widetilde{w}$ is called the nondecreasing rearrangement of $w$ (with respect to the probability $\mu$ ). One may similarly define the nonincreasing rearrangement of $w$ with respect to $\mu$ as the unique right-continuous nonincreasing function which is equimeasurable to $w$.

A crucial feature of rearrangements lies in rearrangement inequalities, essentially discovered by Hardy and Littlewood and extended since. Such inequalities state that integral expressions of the form:

$$
\int_{0}^{\bar{x}} L(v(x), w(x)) d \mu(x)
$$


increase when one replaces the arbitrary functions $v($.$) and w($.$) by their$ nondecreasing rearrangements $\widetilde{v}($.$) and \widetilde{w}($.$) when the integrand L$ satisfies a Spence-Mirrlees type or supermodularity condition (see [Lorentz (1953)]).

Definition 1 Let $L$ be a function $: \mathbb{R} \times \mathbb{R} \rightarrow \mathbb{R} . L$ is called supermodular (respectively strictly supermodular) if, for all $(v, h) \in \mathbb{R} \times \mathbb{R}_{+}^{*}$ :

$w \mapsto[L(v+h, w)-L(v, w)]$ is nondecreasing (respectively increasing) on $\mathbb{R}$.

If $L$ is of class $C^{2}$ a necessary and sufficient condition for $L$ to be supermodular is that the cross-derivative $\partial_{v w}^{2} L$ remains nonnegative. Classical examples of supermodular functions are $L(v, w)=v w, L(v, w)=U(v-w)$ with $U$ concave or $L(v, w)=g(v+w)$ with $g$ convex.

Proposition 2 Let $L$ be a continuous and supermodular function. Let $v$ and $w$ be two real-valued Borel functions defined on $[0, \bar{x}]$ and $\widetilde{v}$ and $\widetilde{w}$ be their nondecreasing rearrangements with respect to $\mu$. We then have:

$$
\int_{0}^{\bar{x}} L(\widetilde{v}(x), \widetilde{w}(x)) d \mu(x) \geq \int_{0}^{\bar{x}} L(v(x), w(x)) d \mu(x),
$$

provided that the rightmost member of this inequality is well-defined.

If $L$ is strictly supermodular, we further have:

Proposition 3 Let $L$ be a continuous and strictly supermodular function. Let $w$ be a real-valued Borel function defined on $[0, \bar{x}]$ and $\widetilde{w}$ be its nondecreasing rearrangement with respect to $\mu$. If one has:

$$
\int_{0}^{\bar{x}} L(x, \widetilde{w}(x)) d \mu(x)=\int_{0}^{\bar{x}} L(x, w(x)) d \mu(x),
$$

then $w=\widetilde{w} \mu$-a.e..

In insurance models, we shall deal with limited liability constraints. The following result gives conditions under which those constraints are closed under rearrangements.

Lemma 4 Let $w$ be any real-valued Borel function defined on $[0, \bar{x}]$ and let $\widetilde{w}$ be its nondecreasing rearrangement with respect to $\mu$. Let $f$ and $g$ be two nondecreasing functions: $[0, \bar{x}] \rightarrow \mathbb{R}$. If $f \leq w \leq g$-a.e, then $f \leq \widetilde{w} \leq g$ $\mu$-a.e.

\subsection{Existence of a solution}

Using rearrangement techniques recalled above, we first obtain: 
Lemma 5 Assume (H1), (H2), (H3) and (MLRC). Let $(w, e) \in A$ and let $\widetilde{w}$ be the nondecreasing rearrangement of $w$ with respect to the probability measure $f(x, e) d x$. Then, $(\widetilde{w}, e) \in A$ and $V(\widetilde{w}, e) \geq V(w, e)$. Moreover the previous inequality is strict unless $w$ is nondecreasing.

Proof: Let $(w, e) \in A$ and let $\widetilde{w}$ be the nondecreasing rearrangement of $w$ with respect to the probability measure $f(x, e) d x$. By equimeasurability, $U(\widetilde{w}, e)=U(w, e) \geq \underline{u}$ and by (MLRC) and Hardy-Littlewood inequality, one has:

$$
\begin{aligned}
& U_{e}(w, e)=\int_{0}^{\bar{x}} u(w(x)) \operatorname{LR}(x, e) f(x, e) d x \\
\leq & \int_{0}^{\bar{x}} u(\widetilde{w}(x)) L R(x, e) f(x, e) d x=U_{e}(\widetilde{w}, e) .
\end{aligned}
$$

This proves that $(\widetilde{w}, e) \in A$.

By strict concavity of $v$, the function $(x, w) \mapsto v(x-w)$ is strictly supermodular. Hence

$$
V(w, e)=\int_{0}^{\bar{x}} v(x-w(x)) f(x, e) d x \leq \int_{0}^{\bar{x}} v(x-\widetilde{w}(x)) f(x, e) d x=V(\widetilde{w}, e)
$$

and by Proposition 3, the inequality is strict unless $w=\widetilde{w}$ a.e..

From Lemma 5, monotonicity is a necessary optimality condition for the doubly-relaxed problem. We may thus assume, without loss of generality, in the doubly-relaxed problem that $w$ is nondecreasing. It should be noted that this monotonicity result is not based on a first-order argument.

Theorem 1 Under assumptions (H1), (H2), (H3) and (MLRC) the doubly-relaxed problem has a solution, $(\bar{w}, \bar{e})$ with $\bar{w}$ nondecreasing on $[0, \bar{x}]$. Moreover, any solution to the doubly-relaxed problem is nondecreasing.

The proof can be found in the appendix.

\section{The second-best problem}

Let us now introduce the condition: 
(CDFC): For all $x \in[0, \bar{x}]$, the function $e \mapsto F(x, e):=\int_{0}^{x} f(s, e) d s$ is convex on $\mathbb{R}_{+}$.

The following concavity result is proved in the appendix.

Lemma 6 Assume (CDFC). If $w$ is nondecreasing and such that $u \circ w$ is integrable on $[0, \bar{x}]$, then the function $e \mapsto U(\bar{w}, e)$ is concave on $\mathbb{R}_{+}$.

The next result is classical, a proof is given in the appendix for the sake of completeness.

Proposition 7 Assume (H1), (H2), (H3), and (MLRC). Let $(\bar{w}, \bar{e})$ be a solution to the doubly-relaxed problem, then:

$$
U_{e}(\bar{w}, \bar{e})=0 .
$$

To be able to solve the second-best problem, we need an additional assumption:

(H4): The problem:

$$
\sup \{V(w, 0):(w, 0) \text { satisfies }(3) \text { and }(4)\}
$$

has a solution $w^{*}$ provided its admissible set is nonempty.

Sufficient conditions for assumption (H4) to be satisfied will be given in Lemmas 8 and 9. Furthermore, we shall see in the next section that, if the principal is risk-neutral, then $(\mathbf{H} 4)$ is satisfied.

We may now state an existence result for the second-best problem:

Theorem 2 Assume (H1), (H2), (H3), (H4), (MLRC) and (CDFC). Then the second-best problem has a solution. Furthermore, we have the following alternative:

- either there exists an optimal solution to the second-best problem of the form $\left(w^{*}, 0\right)$ and $w^{*}$ solves $(7)$,

- or the doubly-relaxed problem and the second-best problem have the same solutions.

\section{Proof:}

If there exists an optimal solution to the second-best problem of the form $\left(w^{*}, 0\right)$ (so that $w^{*}$ solves the program $\left.(7)\right)$ there is nothing to prove. Let us assume then that there exists no second-best solution with $e=0$ and let us prove in this case that the second-best and the doubly-relaxed problems have the same solutions. 
Let $(\bar{w}, \bar{e})$ be a solution to the doubly-relaxed problem (Theorem 1 ensures the existence of such a solution and that $\bar{w}$ is nondecreasing). Lemma 6 and Proposition 7 imply that $(\bar{w}, \bar{e})$ is incentive-compatible. Let us prove that:

$$
\text { if }(w, e) \text { satisfies }(3) \text { and }(4) \text {, then } V(\bar{w}, \bar{e}) \geq V(w, e) \text {. }
$$

Let $(w, e)$ satisfy (3) and (4). If $e>0$, then $(w, e) \in A$, hence $V(\bar{w}, \bar{e}) \geq$ $V(w, e)$. If $e=0$, by $(\mathbf{H} 4)$, there exists a solution $w^{*}$ to $(7)$ and $V\left(w^{*}, 0\right) \geq$ $V(w, 0)$. Since by assumption $\left(w^{*}, 0\right)$ is not a second-best solution, there exists $\left(w^{\prime}, e^{\prime}\right)$ satisfying (3) and (4) with $e^{\prime}>0$ and $V\left(w^{\prime}, e^{\prime}\right)>V\left(w^{*}, 0\right)$. Since $\left(w^{\prime}, e^{\prime}\right) \in A, V(\bar{w}, \bar{e}) \geq V\left(w^{\prime}, e^{\prime}\right)>V(w, 0)$. This proves that $(\bar{w}, \bar{e})$ is a solution to the second-best problem.

Conversely, let $\left(w^{*}, e^{*}\right)$ solve the second-best problem. Since $e^{*}>0,\left(w^{*}, e^{*}\right) \in$ $A$, hence $V\left(w^{*}, e^{*}\right)=V(\bar{w}, \bar{e})$. This establishes that $\left(w^{*}, e^{*}\right)$ solves the doublyrelaxed problem.

Let us now give conditions that ensure that $\mathbf{( H 4 )}$ is satisfied. Proofs are given in the appendix.

Lemma 8 Assume (H1), (H2), (MLRC), (CDFC). If, in addition:

$$
f_{e}(x, 0)=0 \text { for all } x \in[0, \bar{x}],
$$

then $\left(\boldsymbol{H}_{4}\right)$ is satisfied.

Remark. An example of parametrized cumulative distribution functions satisfying (MLRC), (CDFC) and (8) is:

$$
F(x, e):=\left(\frac{x}{\bar{x}}\right)^{\alpha(e)}, \text { for all } e \geq 0
$$

where $\alpha($.$) is any positive convex, differentiable, increasing function such that$ $\alpha^{\prime}(0)=0$

Lemma 9 Assume (H1), (H2), (MLRC). If, in addition, for all $e \geq 0$, the function:

$$
(x, y) \mapsto u(x-y)\left(1-\frac{f(x, e)}{f(x, 0)}\right) \text { is supermodular on }[0, \bar{x}] \times \mathbb{R},
$$

then $\left(\boldsymbol{H}_{4}\right)$ is satisfied. 
Remark. Contrary to condition (8), condition (10) depends on $u$. It is tightly related to the absolute risk aversion index of the agent. Indeed, condition (10) reads as:

$$
-\frac{u^{\prime \prime}(x-y)}{u^{\prime}(x-y)}\left(1-\frac{f(x, e)}{f(x, 0)}\right)+\frac{\partial}{\partial x}\left(\frac{f(x, e)}{f(x, 0)}\right) \geq 0 .
$$

By (MLRC), the second term is nonnegative (see [Milgrom (1981)] for details) whereas the first changes sign and is weighted by risk aversion index. For instance, if $u$ is a CARA function $u(z)=-\exp (-\rho z)$, and if (MLRC) is strengthened to

$$
\frac{\partial}{\partial x}\left(\frac{f(x, e)}{f(x, 0)}\right) \geq \delta>0
$$

then (10) is satisfied if $\rho$ is small enough (or more generally when the risk aversion index is bounded by a small enough constant).

\section{Risk-neutral principal}

In this section we study the case of a risk-neutral principal and of risk-averse agents. More precisely, we assume:

(H1'): The principal is risk neutral i.e. $v(x)=x$ for all $x \in \mathbb{R}$. The agents' utility function $u: \mathbb{R} \rightarrow \mathbb{R}$ is strictly concave, increasing, twice continuously differentiable, and there exists $\alpha \in(0,1)$ such that

$$
\lim _{y \rightarrow+\infty} \frac{u(y)}{y^{\alpha}}=0
$$

We shall see that the linearity of $v$ simplifies the problem since assumption (H4) is always satisfied in this case. As previously, we define the principal's second-best and doubly-relaxed problems. In the risk-neutral case the principal's utility takes the form:

$$
V(w, e):=\int_{0}^{\bar{x}}(x-w(x)) f(x, e) d x .
$$

We first have:

Proposition 10 Under assumptions (H1'), (H2), (H3) and (MLRC) the doubly-relaxed problem has a solution), $(\bar{w}, \bar{e})$ with $\bar{w}$ nondecreasing on $[0, \bar{x}]$. If furthermore (MLRCstrong) is fulfilled, then any solution to the doubly-relaxed problem is nondecreasing. 
The proof is essentially the same as that of Theorem 1. Monotonicity of solutions is no more a necessary optimality condition but, due to linearity, some limiting arguments are simpler. It may be found in the appendix.

The next result is proved in the appendix. It states that $(\mathbf{H} 4)$ is always satisfied in the risk-neutral case.

Lemma 11 Assume (H1'), (H2), (H3) and (MLRC), then:

$$
\sup \{V(w, 0):(w, 0) \text { satisfies (3) and (4) }\}
$$

has a nonincreasing solution $w^{*}$ provided its admissible set is nonempty.

Theorem 3 Assume (H'1), (H2), (H3), (MLRC) and (CDFC). Then the second-best problem has a solution. More precisely, the following holds:

- either there exists an optimal solution to the second-best problem of the form $\left(w^{*}, 0\right)$ and $w^{*}$ solves (11),

- or the doubly-relaxed problem and the second-best problem have the same nondecreasing solutions and any solution $\left(w^{*}, e^{*}\right)$ to the second-best problem is such that $\left(\tilde{w}^{*}, e^{*}\right)$ with $\tilde{w}^{*}$ the nondecreasing rearrangement of $w^{*}$ with respect to $f(x, e) d x$ is a solution to the second-best problem.

Assume furthermore (MLRCstrong), then we have the following alternative:

- either there exists an optimal solution to the second-best problem of the form $\left(w^{*}, 0\right)$ and $w^{*}$ solves (11),

- or the doubly-relaxed problem and the second-best problem have the same solutions.

The proof is similar to that of Theorem 2 and therefore left to the reader.

It is well-known that the drawback of the previous approach is that the CDFC assumption has no obvious economic interpretation. [Jewitt (1988)] proposed alternative conditions under which, in the risk-neutral case, the first-order approach is valid. We mention without proof that our results remain true if one replaces the CDFC assumption by those introduced by Jewitt. 


\section{The case of Insurance}

We end the paper by an application of our method to an insurance framework. Since the main arguments are similar to those given above ${ }^{1}$, proofs are omitted.

\subsection{The model}

An agent with initial wealth $w_{0}$ may incur a random loss $X$ whose density function $f(x, e)$ over the interval $[0, \bar{x}]$ depends on the agent's effort $e$ in avoiding the loss. It is assumed that the agent's utility (which depends on wealth and effort) is von Neumann Morgenstern in wealth and quasi-linear in effort:

$$
U(W, e)=\int_{0}^{\bar{x}} u(W(x)) f(x, e) d x-e .
$$

where $W(x)$ is the agent's wealth if a loss $x$ occurs. The insurer offers a contract $(\Pi, I)$ consisting of an indemnity scheme $I: \mathbb{R}_{+} \rightarrow \mathbb{R}_{+}$such that $0 \leq I(x) \leq x$ and of a premium $\Pi \geq 0$. Whenever he buys a contract, the agent's random wealth is

$$
W(X)=w_{0}-\Pi+I(X)-X .
$$

The agent chooses his effort before uncertainty is revealed subject to achieving a reservation level and an incentive compatibility constraint. The insurer assumed here to be either risk neutral or risk-averse, then chooses the optimal contract so as to maximize his profit. Equivalently he solves the following problem:

$$
\begin{gathered}
\sup _{W, e} V(W, e):=\int_{0}^{\bar{x}} v(-(W(x)+x)) f(x, e) d x \text { subject to : } \\
U(W, e)=\max _{e^{\prime} \geq 0} U\left(W, e^{\prime}\right), \\
U(W, e) \geq \underline{u} \\
e \geq 0, \Pi \geq 0 \\
w_{0}-\Pi-X \leq W(X) \leq w_{0}-\Pi .
\end{gathered}
$$

\subsection{Assumptions}

(H1b): The agent's utility function $u: \mathbb{R} \rightarrow \mathbb{R}$ is strictly concave, increasing and twice continuously differentiable. The insurer's utility function $v: \mathbb{R} \rightarrow \mathbb{R}$

$\overline{1}$ A previous version of the present paper with detailed proofs for the insurance case can be downloaded at: http://www. ceremade.dauphine.fr/Publications.php. 
is concave, increasing and twice continuously differentiable.

(H2b): The density function $f(.,$.$) is continuous on [0, \bar{x}] \times \mathbb{R}_{+}$. For all $x$, $f(x,$.$) is continuously differentiable and the first partial derivatives of f$ with respect to $e, f_{e}$ is jointly continuous on $[0, \bar{x}] \times \mathbb{R}_{+}$.

Let us introduce the relaxed condition:

$$
U_{e}(W, e) \geq 0
$$

(H3b): Let $\mathcal{A}:=\{(\Pi, W, e)$ that satisfies (13), (14), (15), (16) $\}$. Then $\mathcal{A} \neq \emptyset$.

(MLRCb): For all $e \geq 0$, the function $L R(.,):. x \mapsto L R(x, e):=f_{e}(x, e) / f(x, e)$ is nonincreasing on $[0, \bar{x}]$.

(MLRCbstrong): For all $e \geq 0$, the function $L R(.,):. x \mapsto L R(x, e):=$ $f_{e}(x, e) / f(x, e)$ is decreasing on $[0, \bar{x}]$.

(CDFCb): For all $x \in[0, \bar{x}]$, the function $e \mapsto F(x, e):=\int_{0}^{x} f(s, e) d s$ is concave on $\mathbb{R}_{+}$.

As in the previous model, the doubly-relaxed problem is defined by:

$$
\sup \{V(W, e),(\Pi, W, e) \in \mathcal{A}\}
$$

To be able to solve the second-best problem, we need the additional assumption:

(H4b): The problem:

$$
\sup \{V(W, 0),(\Pi, W, 0) \text { satisfies }(12),(13),(14),(15)\}
$$

has a solution provided its admissible set is nonempty.

\subsection{Existence of second-best solutions}

We may now state an existence result for the second-best problem:

Theorem 4 Assume (H1b), (H2b), (H3b), (H4b), (MLRCb) and (CD$\boldsymbol{F C b}$. Then the second-best problem has a solution. More precisely, the following holds: 
- either there exists an optimal solution to the second-best problem of the form $\left(\Pi^{*}, W^{*}, 0\right)$ and $\left(\Pi^{*}, W^{*}\right)$ solves $(17)$,

- or the doubly-relaxed problem and the second-best problem have the same nonincreasing solutions and any solution $(\Pi, W, e)$ to the second-best problem is such that $(\Pi, \tilde{W}, e)$ with $\tilde{W}$ the nonincreasing rearrangement of $W$ with respect to the measure $f(x, e) d x$ is a solution to the second-best problem.

Assume furthermore either (MLRCbstrong) or $v$ strictly concave, then we have the following alternative:

- either there exists an optimal solution to the second-best problem of the form $\left(\Pi^{*}, W^{*}, 0\right)$ and $\left(\Pi^{*}, W^{*}\right)$ solves $(17)$

- or the doubly-relaxed problem and the second-best problem have the same solutions.

Let us finally give sufficient conditions for (H4b) to hold.

Lemma 12 Assume either,

- (H1b), (H2b), (MLRCb), (CDFCb) and $f_{e}(x, 0)=0$ for all $x \geq 0$, or

- (H1b), (H2b), (MLRCb) and that for all $e \geq 0$, the function: $(x, y) \mapsto$ $u(-x+y)\left(1-\frac{f(x, e)}{f(x, 0)}\right)$ is supermodular on $[0, \bar{x}] \times \mathbb{R}$

then $(\boldsymbol{H} \mathbf{4} \boldsymbol{b})$ is satisfied.

\section{$7 \quad$ Appendix}

\subsection{Proof of Theorem 1}

Proof: In what follows, to shorten notations, we shall sometimes write $\int w$ instead of $\int w(x) d x$.

Let us first check that the value of the doubly-relaxed is finite. By (H3), $A \neq \emptyset$. Let $(w, e) \in A$. By assumptions (H1) and (H2), there exists constants $C>0$ and $C^{\prime}>0$ such that

$$
\begin{gathered}
\underline{u} \leq \int_{0}^{\bar{x}} u(w(x)) f(x, e) d x-e \leq C\left(1+\int_{0}^{\bar{x}} w(x) d x\right) \\
V(w, e) \leq C^{\prime}\left(1+\int_{0}^{\bar{x}} x d x-\int_{0}^{\bar{x}} w(x) d x\right) .
\end{gathered}
$$


Hence, by (18), (19) and (H3), the value of the doubly-relaxed problem is finite.

Let us now consider a sequence $\left(w_{n}, e_{n}\right) \in A^{\mathbb{N}}$ such that $V\left(w_{n}, e_{n}\right)$ converges to this finite value. By the estimates (18), (19), and the fact that $e_{n} \geq 0$ we deduce that all the following sequences are bounded:

$$
\int_{0}^{\bar{x}} w_{n}, \int_{0}^{\bar{x}} u\left(w_{n}(x)\right) f\left(x, e_{n}\right) d x, \int_{0}^{\bar{x}} v\left(x-w_{n}(x)\right) f\left(x, e_{n}\right) d x, \text { and } e_{n} .
$$

By Lemma 5, we may assume that each $w_{n}$ is nondecreasing.

Let us now prove that $w_{n}$ is bounded in $L^{1}$. By assumption (H1), there exists constants $b>c>0$ and $b^{\prime}>c^{\prime}>0$ and $\left(a, a^{\prime}\right) \in \mathbb{R}^{2}$ such that for for all $x \in[0, \bar{x}]$ and all $w \in \mathbb{R}$, one has:

$$
u(w) \leq a-b w_{-}+c w_{+}, v(x-w) \leq a^{\prime}-b^{\prime} w_{+}+c^{\prime} w_{-} .
$$

Furthermore, it follows from (20) that there exists $\gamma \in \mathbb{R}$ and $\varepsilon \in(0,1)$ such that:

$$
\begin{aligned}
& \int_{0}^{\bar{x}} w_{n-} \leq \gamma+(1-\varepsilon) \int_{0}^{\bar{x}} w_{n+}, \\
& \int_{0}^{\bar{x}} w_{n+} \leq \gamma+(1-\varepsilon) \int_{0}^{\bar{x}} w_{n-} .
\end{aligned}
$$

Hence the sequence $w_{n}$ is bounded in $L^{1}$. Since each $w_{n}$ is nondecreasing, it follows from Helly's Theorem (see for instance [Natanson (1967)]) that a subsequence of $w_{n}$ (again denoted $w_{n}$ ) converges pointwise to a nondecreasing function $\bar{w}$. Similarly, taking a subsequence if necessary, we may also assume that $e_{n}$ converges to some $\bar{e} \geq 0$.

Let us now prove that $(\bar{w}, \bar{e})$ is a solution to the doubly-relaxed problem. From assumption (H1), there exists a constant $A>0$ such that for all $(x, w) \in$ $[0, \bar{x}] \times \mathbb{R}:$

$$
u(w) \leq A\left(1+|w|^{\alpha}\right), v(x-w) \leq A\left(1+|x-w|^{\alpha}\right) .
$$

Since $\left|w_{n}\right|^{\alpha}$ is bounded in $L^{1 / \alpha}$ which is reflexive, we may assume that $\left|w_{n}\right|^{\alpha}$ converges weakly to $|\bar{w}|^{\alpha}$ in $L^{1 / \alpha}$. This implies in particular that

$$
\lim _{n} \int_{0}^{\bar{x}}\left|w_{n}(x)\right|^{\alpha} f\left(x, e_{n}\right) d x=\int_{0}^{\bar{x}}|\bar{w}(x)|^{\alpha} f(x, \bar{e}) d x .
$$

By Fatou's Lemma, (21) and (22), we obtain

$$
U(\bar{w}, \bar{e}) \geq \varlimsup_{\overline{\lim _{n}}} U\left(w_{n}, e_{n}\right) \geq \underline{u} .
$$


A similar argument yields

$$
V(\bar{w}, \bar{e}) \geq \varlimsup_{n} V\left(w_{n}, e_{n}\right)
$$

Finally, applying Fatou's Lemma and (21) to the sequence $\left(A\left(1+\left|w_{n}\right|^{\alpha}\right)\left|f_{e}\left(x, e_{n}\right)\right|-\right.$ $\left.u\left(w_{n}(x)\right) f_{e}\left(x, e_{n}\right)\right)$, we obtain:

$$
U_{e}(\bar{w}, \bar{e}) \geq 0 .
$$

Hence $(\bar{w}, \bar{e}) \in A$ and from $(23),(\bar{w}, \bar{e})$ is a solution to the doubly-relaxed problem.

The fact that any solution to the doubly-relaxed problem is nondecreasing follows from Lemma 5 .

\subsection{Proof of Lemma 6}

\section{Proof:}

Let us first assume that $w$ is bounded. Since $u \circ w$ is nondecreasing and of bounded variation, the quantity

$$
U(w, e):=\int_{0}^{\bar{x}} u(w(x)) f(x, e) d x-e
$$

can be integrated by parts:

$$
U(w, e):=-\int_{0}^{\bar{x}} F(x, e) d \mu(x)+u(w(\bar{x}))-e
$$

where $\mu$ is a nonnegative measure (the derivative of $u \circ w)$. From the (CDFC) assumption, $F$ is convex with respect to $e$. Hence $e \mapsto U(w, e)$ is concave. This proves that, if $\bar{w}$ is bounded, then $e \mapsto U(\bar{w}, e)$ is concave.

If $w$ is not assumed to be bounded, let us consider the sequence $w_{n}$ defined for $n$ large enough, by:

$$
w_{n}(x)= \begin{cases}w(1 / n) & \text { if } x \in[0,1 / n) \\ w(x) & \text { if } x \in[1 / n, \bar{x}-1 / n] \\ w(\bar{x}-1 / n) & \text { if } x \in[\bar{x}-1 / n, \bar{x}] .\end{cases}
$$


By the preceding, argument, for all $n$, the function $e \mapsto U\left(w_{n}, e\right)$ is concave. By Lebesgue's Dominated Convergence Theorem and the fact that $u(w)$ is in $L^{1}$, for every $e \geq 0$ :

$$
\lim _{n} U\left(w_{n}, e\right)=U(w, e)
$$

which proves the desired concavity result.

\subsection{Proof of Proposition 7}

Proof: $\quad$ Let us first prove that $U_{e}(\bar{w}, \bar{e})=0$. We already know that $U_{e}(\bar{w}, \bar{e}) \geq$ 0 . The Lagrangian of the doubly-relaxed problem is:

$$
\mathcal{L}(w, e, \lambda, \mu, \nu):=V(w, e)+\lambda e+\mu U(w, e)+\nu U_{e}(w, e)
$$

Kuhn and Tucker necessary conditions (it is easy to check that the constraints of the doubly-relaxed problem are qualified at $(\bar{w}, \bar{e})$ ) yield that for nonnegative multipliers $(\lambda, \mu, \nu)$ :

$$
\begin{gathered}
V_{e}(\bar{w}, \bar{e})+\lambda+\mu U_{e}(\bar{w}, \bar{e})+\nu U_{e e}(\bar{w}, \bar{e})=0 \\
v^{\prime}(x-\bar{w}(x))=\mu u^{\prime}(\bar{w}(x))+\nu u^{\prime}(\bar{w}(x)) L R(x, \bar{e})
\end{gathered}
$$

Assume now by contradiction that $U_{e}(\bar{w}, \bar{e})>0$. Then the complementary slackness condition in Kuhn and Tucker Theorem implies that $\nu=0$ and (25) yields

$$
v^{\prime}(x-\bar{w}(x))=\mu u^{\prime}(\bar{w}(x))
$$

so that $\mu>0$ and $x \mapsto x-\bar{w}(x)$ is nondecreasing. Now (24) implies:

$$
V_{e}(\bar{w}, \bar{e}) \leq-\mu U_{e}(\bar{w}, \bar{e})<0
$$

As

$$
V_{e}(\bar{w}, \bar{e})=\int_{0}^{\bar{x}} v(x-\bar{w}(x)) L R(x, e) f(x, e) d x
$$

and as the correlation with respect to the probability measure $f(x, e) d x$ of the two nondecreasing functions $v(x-\bar{w}(x))$ and $L R(x, e)$ is nonnegative, we have

$$
V_{e}(\bar{w}, \bar{e}) \geq \int_{0}^{\bar{x}} v(x-\bar{w}(x)) f(x, e) d x \int_{0}^{\bar{x}} L R(x, e) f(x, e) d x .
$$

As the second factor in the rightmost member of this inequality is zero, we get a contradiction with $(26)$. Hence $U_{e}(\bar{w}, \bar{e})=0$. 


\subsection{Proof of Lemma 8}

Proof: If the admissible set of (7) is empty, there is nothing to prove. Let $w$ be admissible for $(7)$ and $\widetilde{w}$ be the nondecreasing rearrangement of $w$ with respect to $f(x, 0) d x$. As in the proof of Theorem 1 , one has, $V(\widetilde{w}, 0)>V(w, 0)$ and $U(\widetilde{w}, 0)=U(w, 0) \geq \underline{u}$. Let us check that $(\widetilde{w}, 0)$ is incentive-compatible. By Lemma 6,

$$
e \mapsto U(\widetilde{w}, e) \text { is concave on } \mathbb{R}_{+}
$$

and from $(8), U_{e}(\widetilde{w}, 0)=-1$. Thus $(\widetilde{w}, 0)$ is incentive-compatible. hence monotonicity is a necessary optimality condition for (7). The existence proof may then be done exactly as in the proof of theorem 1 .

\subsection{Proof of Lemma 9}

Proof: If the admissible set of (7) is empty there is nothing to prove. Let $w$ be such that $(w, 0)$ satisfies $(3)$ and $(4)$ and let

$$
\hat{w}(x):=x-\widetilde{y}(x)
$$

where $y(x):=x-w(x)$ and $\widetilde{y}$ denotes the nondecreasing rearrangement of $y$ with respect to $f(x, 0) d x$. By equimeasurability of $x-\hat{w}$ and $x-w$, we first have $V(\hat{w}, 0)=V(w, 0)$. From $(6)$, on the one hand, we obtain:

$$
U(\hat{w}, 0) \geq U(w, 0) \geq \underline{u} .
$$

On the other hand, from (3) and (10), we have that, for all $e \geq 0$ :

$$
\begin{aligned}
\int_{0}^{\bar{x}} u(\hat{w}(x))(f(x, 0)-f(x, e)) d x & =\int_{0}^{\bar{x}} u(x-\widetilde{y}(x))\left(1-\frac{f(x, e)}{f(x, 0)}\right) f(x, 0) d x \\
& \geq \int_{0}^{\bar{x}} u(w(x))(f(x, 0)-f(x, e)) d x \geq-e .
\end{aligned}
$$

Hence $(\hat{w}, 0)$ is incentive-compatible.

The previous argument shows that there exists a a maximizing sequence $\left(w_{n}\right)$ of (7) such that, for every $n, x \mapsto x-w_{n}(x)$ is nondecreasing and we end the proof, by the same estimates and convergence arguments as in Theorem 1 (applying Helly's theorem to $x-w_{n}$ which does not change the arguments of the proof). 


\subsection{Proof of Proposition 10}

Proof: We still denote by $A$ the admissible set of the doubly-relaxed problem. By (H3), $A \neq \emptyset$. Let $(w, e) \in A$. By assumptions (H1') and (H2) there exists a constant $C>0$ such that

$$
\underline{u} \leq \int_{0}^{\bar{x}} u(w(x)) f(x, e) d x-e \leq C\left(1+\int_{0}^{\bar{x}} w(x) d x\right) .
$$

By linearity of $V$, there exists a constant $C^{\prime}$ such that

$$
V(w, e) \leq C^{\prime} \text { for all }(w, e) \in A .
$$

Hence, by (28) and (H3), the value of the doubly-relaxed problem is finite.

Let us now consider a sequence $\left(w_{n}, e_{n}\right) \in A^{\mathbb{N}}$ such that $V\left(w_{n}, e_{n}\right)$ converges to this finite value. By the estimates (27), (28), and the fact that $e_{n} \geq 0$, we deduce that the following sequences are bounded:

$$
\int_{0}^{\bar{x}} w_{n}, \int_{0}^{\bar{x}} u\left(w_{n}(x)\right) f\left(x, e_{n}\right) d x, V\left(w_{n}, e_{n}\right), \text { and } e_{n} .
$$

By Lemma 5, we may also assume that each $w_{n}$ is nondecreasing.

Let us now prove that $w_{n}$ is bounded in $L^{1}$. By assumption (H1'), there exists constants $a \in \mathbb{R}$ and $b>c>0$ such that for all $x \in[0, \bar{x}]$ and all $w \in \mathbb{R}$, one has:

$$
u(w) \leq a-b w_{-}+c w_{+} .
$$

Hence, it follows from (29) that there exists $\gamma \in \mathbb{R}$ and $\varepsilon \in(0,1)$ such that:

$$
\int_{0}^{\bar{x}} w_{n+} \geq \gamma+(1+\varepsilon) \int_{0}^{\bar{x}} w_{n-} .
$$

Thus:

$$
\int_{0}^{\bar{x}} w_{n}=\int_{0}^{\bar{x}} w_{n+}-\int_{0}^{\bar{x}} w_{n-} \geq \gamma+\varepsilon \int_{0}^{\bar{x}} w_{n-}
$$

since $\int_{0}^{\bar{x}} w_{n}$ is bounded so are $\int_{0}^{\bar{x}} w_{n-}$ and $\int_{0}^{\bar{x}} w_{n+}$. This shows that $w_{n}$ is bounded in $L^{1}$. Since each $w_{n}$ is nondecreasing, some subsequence of $w_{n}$ (again denoted $w_{n}$ ) converges pointwise to a nondecreasing function $\bar{w}$. Similarly, taking a subsequence if necessary, we may also assume that $e_{n}$ converges to $\bar{e} \geq 0$. 
Since $w_{n}$ is bounded in $L^{1}$ (again up to a subsequence) we may assume that the sequence of Radon measures $\mu_{n}$ defined by $d \mu_{n}:=w_{n} d x$ converges weakly $*$ (in the sense of the duality between continuous functions and Radon measures) to some measure $\mu$. Since $w_{n}$ converges pointwise to $\bar{w}$, one obviously has $d \mu=\bar{w} d x$ and since $f\left(x, e_{n}\right)$ converges uniformly to $f(x, \bar{e})$, we get:

$$
\lim \int_{0}^{\bar{x}} w_{n}(x) f\left(x, e_{n}\right) d x=\int_{0}^{\bar{x}} f(x, \bar{e}) d \mu(x)=\int_{0}^{\bar{x}} \bar{w}(x) f(x, \bar{e}) d x .
$$

This implies that:

$$
\lim _{n} V\left(w_{n}, e_{n}\right)=V(\bar{w}, \bar{e})
$$

Finally we prove exactly as in the proof of Theorem 1 that

$$
U(\bar{w}, \bar{e}) \geq \varlimsup_{\overline{\lim }} U\left(w_{n}, e_{n}\right) \geq \underline{u} \text { and } U_{e}(\bar{w}, \bar{e}) \geq 0 .
$$

Hence $(\bar{w}, \bar{e}) \in A$ and from (30) we deduce that $(\bar{w}, \bar{e})$ is a solution to the doubly-relaxed problem.

It remains to prove the second assertion under (MLRCstrong). Assume that $(w, e)$ is a solution to the doubly-relaxed problem, then so is $(\widetilde{w}, e)$ (where $\widetilde{w}$ is the nondecreasing rearrangement of $w$ with respect to the probability measure $f(x, e) d x)$. By the same arguments as in the proof of Proposition 7 , one has $U_{e}(\widetilde{w}, e)=0$. On the other hand, by Proposition 3, (MLRCstrong) implies $U_{e}(\widetilde{w}, e) \geq U_{e}(w, e) \geq 0$ and the inequality is strict unless $w=\widetilde{w}$ a.e., so that $w$ is nondecreasing.

\subsection{Proof of Lemma 11}

Proof: If the admissible set of (11) is empty, there is nothing to prove. Let us therefore assume that it is nonempty. Let $w$ be such that $(w, 0)$ satisfies (3) and (4), and let $\hat{w}$ be the nonincreasing rearrangement of $w$ with respect to $f(x, 0) d x$. By equimeasurability, one first gets:

$$
U(\hat{w}, 0)=U(w, 0) \geq \underline{u} \text { and } V(\hat{w}, 0)=V(w, 0)
$$

Let us prove that $(\hat{w}, 0)$ is incentive-compatible. For all $e>0$, from (MLRC) one has $x \mapsto \frac{f(x, e)}{f(x, 0)}$ is nondecreasing. Hence, by Hardy-Littlewood's inequality, 
we get:

$$
\begin{array}{r}
\int_{0}^{\bar{x}} u(\hat{w}(x))\left(1-\frac{f(x, e)}{f(x, 0)}\right) f(x, 0) d x \\
\geq \int_{0}^{\bar{x}} u(w(x))\left(1-\frac{f(x, e)}{f(x, 0)}\right) f(x, 0) d x \geq 0,
\end{array}
$$

which proves that $(\hat{w}, 0)$ is incentive-compatible. The existence proof may then be done exactly as in the proof of theorem 1.

\section{References}

[Balder (1996)] E. Balder, On the existence of optimal contract mechanisms for incomplete information principal-agent models, J. Econ. Theory, 68 (1996), no. 1, $133-148$.

[Carlier and Dana (2002)] G. Carlier and R.-A. Dana, Rearrangement inequalities in nonconvex economic models, cahier du CEREMADE 0238.

[Grossman and Hart (1983)] S. Grossman and O. Hart, An analysis of the principalagent problem, Econometrica 51 (1983), no. 1, 7-45.

[Hardy et al. (1988)] G. H. Hardy, J. E. Littlewood and G. Pòlya, Inequalities, Reprint of the 1952 edition, Cambridge University Press, Cambridge, (1988).

[Holmstrom (1975)] B. Holmstrom, Moral hazard and observability, Bell journal of Economics 10 (1975), 74-91.

[Jewitt (1988)] I. Jewitt, Justifying the first-order approach to principal-agent problems Econometrica 56 (1988), no. 5, 1177-1190.

[Lorentz (1953)] G.G. Lorentz, An inequality for rearrangements, Amer. Math. Monthly 60 (1953), 176-179.

[Milgrom (1981)] P. Milgrom, Good News and Bad News: Representation Theorems and Applications, Bell Journal of Economics, 12 (1981), no. 2, 380-391.

[Mirrlees (1975)] J. Mirrlees, The theory of Moral hazard and unobservable behaviorPart1 Mimeo, Nuffield College, Oxford, (1975).

[Natanson (1967)] I.P. Natanson, Theory of functions of a real variable, Mc GrawHill, (1967).

[Page (1987)] F. Page, The existence of optimal contracts in the principal-agent model, J. Math. Econom. 16 (1987), no. 2, 157-167. 
[Page (1991)] F. Page, Optimal contract mechanisms for principal-agent problems with moral hazard and adverse selection, Econ. Theory 1 (1991), no. 4, 323-338.

[Rogerson (1985)] W.P. Rogerson, The first-order approach to principal-agent problems, Econometrica 53 (1985), no. 6, 1357-1367. 